\title{
Formación docente en Educación Especial: hábitos de estudio y práctica docente
}

\section{Teacher training in special education: study habits and teaching practice}

Dr. Mauricio Zacarías Gutiérrez es Profesor-Investigador de la Escuela Normal Fray Matías de Córdova, ubicada en Tapachula, Chiapas (México), (mazag@hotmail.com) (http://orcid.org/0000-0003-4564-5673)

Ma. Juana Eva Luna Denicia es Profesora-Investigadora de la Escuela Normal Fray Matías de Córdova, ubicada en Tapachula, Chiapas (México), (evacarton@hotmail.com) (http://orcid.org/0000-0002-0830-7731)

Recibido: 2017-07-25 / Revisado: 2017-08-28 / Aceptado: 2017-11-01 / Publicado: 2018-07-01

\section{Resumen}

La presente investigación se llevó a cabo con estudiantes de la licenciatura en Educación Especial de la escuela normal Fray Matías de Córdova, ubicada en la ciudad de Tapachula de Córdova y Ordóñez, Chiapas; México. El objetivo del artículo es dar cuenta de los hábitos de estudio que poseen los estudiantes y la opinión que tienen de la práctica docente que realizan los profesores que los forman. Los resultados que aquí se presentan se recuperaron a través de un cuestionario aplicado a $|0|$ estudiantes matriculados en el semestre agosto 2015-enero 2016. Se encontró que estos estudiantes tienen como hábitos de estudio principalmente realizar resúmenes y esquemas para apropiarse del contenido académico que señala la antología - compilación de lecturas elaborada por un equipo de académicos o por el profesor de una asignatura - nombran a la vez, que el espacio físico de la casa es el lugar donde especialmente realizan el proceso de atender las tareas escolares; respecto a la opinión que tienen de la práctica docente de los profesores, indican que la exposición del estudiante, la participación individual, la entrega de trabajos y el examen escrito son prácticas cotidianas en los profesores que los forman. Se concluye, que los hábitos de estudios y las prácticas docentes de los profesores formadores se imbrican en la formación de docentes en educación especial.

Descriptores: Docencia, educación especial, escuela, hábito, práctica docente, profesión

\begin{abstract}
The present research was carried out with students of the degree in Special Education of the normal school Fray Matías de Córdova, located in the city of Tapachula de Córdova and Ordóñez, Chiapas; Mexico. The objective of the article is to give an account of the study habits that the students have and the opinion they have of the teaching practice that the professors
\end{abstract}

Forma sugerida de citar: Zacarías, M., \& Luna, Ma. J. (2018). Formación docente en Educación Especial: hábitos de estudio y práctica docente. Alteridad, 13(2), 262-273. https://doi.org/10.17163/alt.v13n2.2018.09. 
that form them perform. The results presented here were retrieved through a questionnaire applied to $|0|$ students enrolled in the semester August 20 I5-January 2016. It was found that these students have as study habits mainly the realization of summaries and graphic organizers to appropriate the academic content of the anthology, which is a compilation of readings elaborated by a team of academics or by the teacher of a subject. At the same time, it was also found that the physical space of the house is the place where they especially perform the process of attending school tasks; As for

\section{Introducción}

La investigación se llevó a cabo con estudiantes de la licenciatura en Educación Especial de la Escuela Normal Fray Matías de Córdova, ubicada en la ciudad de Tapachula de Córdova y Ordóñez, Chiapas, inscritos en el semestre agosto 2015-enero 2016. El artículo tiene como objetivo analizar los hábitos de estudio de los estudiantes y la opinion que tienen respecto a la práctica docente de los profesores que los forman. Se aplicó un cuestionario a 101 de 104 estudiantes que conformaban el 100\% de la matrícula escolar en ese momento.

Cabe contextualizar que en México desde principios del siglo XIX se inicia con la atención a la Educación Especial, sin embargo, la primera institución formadora de docentes en el campo de la educación especial fue creada en 1867, iniciando con la escuela normal de Sordo-Mudos (Plan de Estudios, 2004; Secretaría de Educación Pública, 2010). Fue hasta 1970 cuando se crea la Dirección General de Educación Especial, que regularía el servicio a las necesidades educativas en todo el país (Secretaría de Educación Pública, 2006). Mas, el proceso de atender la educación especial en las entidades del país ha sido lenta, por ejemplo, "en la década de los 90 los estados de: Colima, Chiapas, Chihuahua, Guanajuato, Michoacán, Morelos y Tlaxcala" (Plan de Estudios, 2004, p. 20), no tenían en ninguna escuela normal la licenciatura en Educación Especial.

En Chiapas, la formación docente en Educación Especial inicia en 2004, ofertándose the opinion students have of the teaching practice of the teachers, they pointed out that the student's oral presentations, the individual participation, the delivery of works and the written exam are daily practices in the professors that form them. It is concluded that the study habits and the teaching practices of the teacher trainers are interwoven in the training of teachers in special education.

Keywords: Habit, school, special education, profession, teaching, teaching practice

en la Escuela Normal Fray Matías de Córdova, ubicada en la ciudad de Tapachula de Córdova y Ordóñez; Chiapas, y en el año 2007 en la Escuela Normal de Licenciatura en Educación Primaria del Estado, ubicada en la ciudad de Tuxtla Gutiérrez. Por tanto, la atención a la poblacion estudiantil con necesidades educativas especiales en edad de cursar la educación básica en la entidad, hasta antes que las escuelas normales ofertaran la licenciatura en Educación Especial, se cubrió con egresados de las licenciaturas de Educación Primaria, Preescolar, licenciados en Educación Especial formados en otras entidades, psicólogos en sus diversas terminales, entre otros. La licenciatura en Educación Especial se imparte en las escuelas normales del país, y atienden el plan y programa de estudio que establece la Secretaría de Educación Pública. Es la única institución facultada por el artículo Tercero Constitucional, para determinar los planes y programas de estudio para la formación del profesorado de educación básica (Constitución Política de los Estados Unidos Mexicanos, 2016).

Para problematizar el estudio de la formación docente en Educación Especial, se revisó el planteamiento del Plan Nacional de Desarrollo (2013), el Programa Sectorial de Educación (2013), la Ley General de Educación (2013), entre otros. No obstante, la formación docente para la educación especial ha sido objeto de investigación. En este caso, se exploró el planteamiento de Sánchez-Palomino (2007), Farías-Martínez y Ramírez-Montoya (2010), Gallego-Ortega y 
Rodríguez-Fuentes (2007) y Tenorio (2011), quienes desde sus propias miradas de abordar la formación inicial docente y la atención a las necesidades educativas especiales permiten profundizar en el cuestionamiento del proceso formativo de los estudiantes que participaron en esta pesquisa.

Así mismo, se indagó respecto al desarrollo de habilidades académicas en el estudiante para atender el contenido académico, puesto que se aspira a través del trabajo científico, formarles un conocimiento que los diferencie de otros campos disciplinares, de tal manera, que los involucrados lo mantegan o modifiquen, por consiguiente, la pertenencia al grupo, en este caso la escuela, asegura la defensa de los intereses de sus miembros (Bourdieu, 2009). En tal caso, el estudiante, en función de sus hábitos de estudio atiende de manera explícita e implícita las implicaciones de la formación, forjándose así un trayecto formativo, pues "la ejercitación, la práctica y la automatización de las destrezas son importantes para los estudiantes, especialmente para aquellos con dificultades de aprendizaje" (Torres, Tolosa, Urrea y Monsalve, 2009); sin embargo, estas acciones le implican al docente, el conocimiento de los hábitos de estudio de los estudiantes, para implementar las estrategias didácticas que favorezcan el aprendizaje (Escalante, Escalante, Linzaga y Merlos, 2008).

El planteamiento de Escalante, Escalante, Linzaga y Merlos (2008), respecto a que es necesario que el docente conozca los hábitos de estudio del estudiante con el fin de llevarlo al éxito académico, se hila con el trabajo de Álvarez, Silió y Fernández (2012), quienes destacan que la planificación, colaboración e innovación son piezas claves para conseguir una buena práctica educativa, pues a través de ella se cuestiona y se provoca la confrontación del conocimiento implícito, explorando las vivencias de los alumnos. Al respecto Chirinos y Padrón (2010) sostienen que la práctica docente está vinculada con la disposición del estudiante por aprender, mas el profesor, es el que tiene que motivar para el logro, buscando con ello el enfrentamiento a las diferentes realidades de los estudiantes de una manera comprometida; las estrategias docentes deben estar dirigidas a conseguir equidad y justicia en el hecho educativo.

La contextualización anterior, problematizó la formación académica de los estudiantes de la licenciatura en Educación Especial de la escuela normal Fray Matías de Córdova. Por tal, se plantea la pregunta de investigación, ¿qué hábitos de estudio tienen los estudiantes que cursan la licenciatura y qué opinan de la práctica docente de los profesores que los forman?. Teniendo como objetivo, analizar los hábitos de estudio que poseen y la opinión que se han hecho de la práctica docente de los profesores que los forman; y sosteniendo la siguiente hipótesis: los hábitos de estudio de los estudiantes están en sinergia con las prácticas docentes del profesorado que los forma.

\section{Metodología}

El estudio se realizó con alumnos de la licenciatura en Educación Especial de la Escuela Normal Fray Matías de Córdova, ubicada en la ciudad de Tapachula de Córdova y Ordóñez, Chiapas; matriculados en el semestre agosto 2015-enero 2016. Se trabajó con 101 alumnos de 104 que componían la matrícula escolar en ese momento: 29 estudiantes en primer semestre; 22 estudiantes en tercer semestre; 27 estudiantes en quinto semestre; 26 estudiantes en séptimo semestre. Cabe decir que esta licenciatura se ofrece anualmente en el turno matutino, por lo que el inicio del semestre non se da en el mes de agosto y el del semestre par en febrero. El diseño de la investigación es transversal descriptivo (Bericat, 1998; Campos, 2014; Ortiz y García, 2016). Para ingresar a la escuela normal se obtuvo el permiso del director de la misma.

Se aplicó un cuestionario para obtener información, el tiempo utilizado fue de tres horas. Se ocuparon cuatro días, un día por cada semestre (primero, tercero, quinto y séptimo); de cada día se ocuparon las primeras horas del inicio laboral -45 minutos con cada grupo-, esto con el fin de evitar fatiga en los estudiantes. 
El cuestionario se aplicó de manera censal, por lo que un único criterio que consideró la elección no probabilística de la muestra, fue que el estudiante estuviera matriculado en ese periodo. El cuestionario estuvo diseñado por los autores en función de los objetivos y la hipótesis de la investigación, consideró las siguientes categorías: datos generales; datos socioeconómicos; principal fuente de información; lugar para realizar tareas; apuntes que solicitan los profesores; estrategia didáctica del profesor; correcciones y comentarios de los trabajos; criterios de evaluación que utiliza.

Los datos obtenidos del cuestionario se depositaron al programa estadístico SPSS, en el cual se ordenaron y codificaron. Con la base de datos se hizo análisis de frecuencias.

\section{Resultados}

En el semestre agosto 2015-enero 2016, se matricularon 104 estudiantes en la licenciatura en Educación Especial. En el momento de aplicar el cuestionario tres estudiantes no estuvieron presentes, por lo que solo se muestra la respuesta de 101 estudiantes.

Referente a la cantidad de estudiantes que estaba cursando la licenciatura, $79,2 \%$ correspondían al sexo femenino y $20,8 \%$ al sexo masculino. En cuanto a la edad, oscilaban entre los 18 a 23 años. El 29,7\% se encontraba en los 21 años (ver tabla 1).

Tabla 1. Edad de los estudiantes que cursan la licenciatura en Educación Especial. Ciclo escolar agosto 2015-enero 2016

\begin{tabular}{|c|c|c|}
\hline Edad & Frecuencia & Total \% \\
\hline 18 & 19 & 18,8 \\
\hline 19 & 19 & 18,8 \\
\hline 20 & 20 & 19,8 \\
\hline 21 & 30 & 29,7 \\
\hline 22 & 12 & 11,9 \\
\hline 23 & 1 & 1,0 \\
\hline Total & 101 & 100,0 \\
\hline
\end{tabular}

Fuente: elaboración propia

Cuando se preguntó a los estudiantes sobre la principal fuente de información que acostumbran a consultar para atender los con- tenidos de las asignaturas, comentaron que es la antología propuesta por el plan y programa de estudio (ver tabla 2).

Tabla 2. Principal fuente de lectura que tienen los estudiantes de la licenciatura en Educación Especial para la formación académica

\begin{tabular}{|l|l|l|l|l|l|l|}
\hline \multicolumn{1}{|c|}{ Fuentes de información } & No contestó & Siempre & Casi siempre & Casi nunca & Nunca & Total \% \\
\hline Antologías & 0,0 & 79,2 & 18,8 & 1,0 & 1,0 & 100 \\
\hline Bibliografía del programa & 2,0 & 33,7 & 39,6 & 21,8 & 3,0 & 100 \\
\hline Bibliografía que busco por mi cuenta & 1,0 & 25,7 & 46,5 & 25,7 & 1,0 & 100 \\
\hline
\end{tabular}

Fuente: elaboración propia 
La tabla muestra que la antología que establece el programa de estudio es la principal fuente de información de los estudiantes: el 79,2\% la ubican como siempre y el 18,8\% como casi siempre. En seguida están las bibliografías complementarias que establece el programa de estudio $(33,7 \%)$ y la bibliografía que ocupan por su cuenta $(25,7 \%)$.

Tabla 3. Lugares donde el estudiante de la licenciatura de Educación Especial realiza tareas escolares

\begin{tabular}{|l|l|l|l|l|l|l|}
\hline Lugar de realizar tareas & No contestó & \multicolumn{1}{|c|}{ Siempre } & Casi siempre & Casi nunca & Nunca & Total \% \\
\hline Casa & 1,0 & 87,1 & 6,9 & 1,0 & 4,0 & 100 \\
\hline Aula de clase & 6,9 & 31,7 & 40,6 & 14,9 & 5,9 & 100 \\
\hline Biblioteca & 5,9 & 7,9 & 36,6 & 43,6 & 5,9 & 100 \\
\hline
\end{tabular}

Fuente: elaboración propia

La antología está cargada de teoría, la cual atiende el propósito de cada asignatura para la formación inicial docente. Con base en ello y en el proceso de desentrañar cómo se apropian
Al preguntarle a los estudiantes sobre los lugares dónde realizan sus tareas académicas, se encontró que el principal lugar es la casa, 94\% estudiantes en total — sumando siempre y casi siempre- hicieron este señalamiento, seguido del aula de clase y finalmente la biblioteca (ver tabla 3$)$.

Tabla 4. Estilos de apuntes que los docentes solicitan al estudiante de la licenciatura en Educación Especial después de realizar lecturas

\begin{tabular}{|l|l|l|l|l|}
\hline \multicolumn{1}{|c|}{ Apuntes solicitados } & No contestó & \multicolumn{1}{c|}{ Sí } & No & Total \% \\
\hline Resúmenes & 1,0 & 94,1 & 5,0 & 100 \\
\hline Diagramas & 5,9 & 59,4 & 34,7 & 100 \\
\hline Esquemas & 3,0 & 79,2 & 17,8 & 100 \\
\hline Cuestionarios & 6,9 & 53,5 & 39,6 & 100 \\
\hline Notas & 7,9 & 61,4 & 30,7 & 100 \\
\hline Subrayado & 0,0 & 100 & 0,0 & 100 \\
\hline
\end{tabular}

Fuente: elaboración propia

La información que presenta la tabla deja ver dos cuestiones, que la principal actividad que el estudiante realiza de la lectura es subrayar: el $100 \%$ de estudiantes indicaron la respuesta, seguido de elaborar resúmenes $(94,1 \%)$ y esquemas $(79,2 \%)$. Estas actividades de apropiación del contenido académico en los estudiantes permite inferir que después de leer —el subrayado va inmerso en el proceso de lectura- se plasman las ideas a través de un control de lectura. los estudiantes de la información que plantea la teoría que leen, se planteó el cuestionamiento ¿cuáles son los principales controles de lectura que los docentes solicitan? (ver tabla 4). 
Tabla 5. Principal estrategia didáctica ocupada por el profesor en el proceso formativo del estudiante de la licenciatura en Educación Especial

\begin{tabular}{|l|l|l|l|l|l|l|}
\hline \multicolumn{1}{|c|}{ Estrategia didáctica } & \multicolumn{1}{c|}{$\begin{array}{c}\text { No } \\
\text { contestó }\end{array}$} & \multicolumn{1}{|c|}{ Siempre } & $\begin{array}{c}\text { Casi } \\
\text { siempre }\end{array}$ & $\begin{array}{c}\text { Casi } \\
\text { nunca }\end{array}$ & Nunca & Total\% \\
\hline Exposición docente & 0,0 & 24,8 & 48,5 & 6,9 & 19,8 & 100 \\
\hline Dictado & 1,0 & 5,0 & 23,8 & 37,6 & 32,7 & 100 \\
\hline Dinámicas de grupo & 0,0 & 48,5 & 45,5 & 2,0 & 4,0 & 100 \\
\hline Exposición de estudiantes & 0,0 & 75,2 & 22,8 & 1,0 & 1,0 & 100 \\
\hline Intervención de estudiantes en clase & 1,0 & 53,5 & 38,6 & 5,0 & 2,0 & 100 \\
\hline Cuestionamientos generados por el profesor & 0,0 & 74,3 & 24,8 & 0,0 & 1,0 & 100 \\
\hline
\end{tabular}

Fuente: elaboración propia

Los resultados que presenta la tabla, solo de la opción siempre, da cuenta que la principal actividad didáctica de los profesores es que los estudiantes expongan en clase $(75,2 \%)$, seguido del cuestionamiento del profesor hacia los mismos $(74,3 \%)$, y de la intervención - participación- del estudiante a partir del contenido teórico y/o empírico de la lectura que esté analizando. Esto permite reconocer que la práctica docente del profesor formador, tiene a la antología como principal herramienta de trabajo. En la tabla 2, se mostró que el estudiante indicó a la antología como la principal fuente de estudio.

Otro elemento que se consideró, fue la revisión de las actividades académicas solicitadas - tareas-, por lo que se planteó a los estudiantes si los profesores regresaban los trabajos solicitados con correcciones y comentarios. En este caso, el $39,6 \%$ de estudiantes mencionaron que siempre y el $34,7 \%$ que casi siempre devuelven los trabajos académicos solicitados con correcciones y comentarios, sin embargo, el $24,8 \%$ de estudiantes afirmaron que los profeso- res casi nunca regresan los trabajos que solicitan de tareas escolares, solo el $1 \%$ dijo que nunca devuelven los trabajos.

De igual manera, las prácticas docentes que los estudiantes viven por parte de los profesores que los forman contribuyen a su formación, en este sentido, se preguntó sobre la presentación del programa de estudio y las formas acordadas de evaluación que tienen los profesores: el 71,3\% precisó que los profesores siempre al inicio del semestre presentan el programa, y el $74,3 \%$ mencionó que siempre respetan las formas de evaluación acordadas.

En relación a la idea anterior, se cuestionó a los estudiantes sobre los criterios que usan los profesores formadores para la asignación de calificaciones, se consideraron los siguientes ítems: examen escrito, examen oral, controles de lectura, trabajos, participación en clase, asistencia, puntualidad, conducta, exposición individual, exposición grupal, planos y maquetas, prácticas de campo, autoevaluación, evaluación colectiva (ver tabla 6).

Tabla 6. Principales criterios que los profesores establecen a los estudiantes para acreditar la asignatura

\begin{tabular}{|l|l|l|l|l|l|l|}
\hline \multicolumn{1}{|c|}{ Criterios de calificación } & \multicolumn{1}{|c|}{ Siempre } & $\begin{array}{c}\text { Casi } \\
\text { siempre }\end{array}$ & \multicolumn{1}{c|}{$\begin{array}{c}\text { Casi } \\
\text { nunca }\end{array}$} & \multicolumn{1}{c|}{ Nunca } & \multicolumn{1}{c|}{$\begin{array}{c}\text { No } \\
\text { contestó }\end{array}$} & \multicolumn{1}{c|}{ Total \% } \\
\hline Examen escrito & 90,1 & 7,9 & 1,0 & 0,0 & 1,0 & 100 \\
\hline Examen oral & 22,8 & 20,8 & 36,6 & 16,8 & 3,0 & 100 \\
\hline Control de lectura & 68,3 & 17,8 & 5,9 & 5,0 & 3,0 & 100 \\
\hline Trabajos & 84,2 & 11,9 & 2,0 & 0,0 & 2,0 & 100 \\
\hline
\end{tabular}




\begin{tabular}{|l|l|l|l|l|l|l|}
\hline \multicolumn{1}{|c|}{ Criterios de calificación } & \multicolumn{1}{|c|}{ Siempre } & $\begin{array}{c}\text { Casi } \\
\text { siempre }\end{array}$ & $\begin{array}{c}\text { Casi } \\
\text { nunca }\end{array}$ & \multicolumn{1}{|c|}{ Nunca } & \multicolumn{1}{c|}{$\begin{array}{c}\text { No } \\
\text { contestó }\end{array}$} & \multicolumn{1}{c|}{ Total \% } \\
\hline Participación en clase & 86,1 & 11,9 & 1,0 & 0,0 & 1,0 & 100 \\
\hline Asistencia & 70,3 & 18,8 & 5,9 & 3,0 & 2,0 & 100 \\
\hline Puntualidad & 61,4 & 25,7 & 7,9 & 2,0 & 3,0 & 100 \\
\hline Conducta & 34,7 & 39,6 & 14,9 & 6,9 & 4,0 & 100 \\
\hline Exposición individual & 76,2 & 19,8 & 2,0 & 0,0 & 2,0 & 100 \\
\hline Exposición grupal & 78,2 & 17,8 & 3,0 & 0,0 & 1,0 & 100 \\
\hline Planos y maquetas & 30,7 & 45,5 & 18,8 & 3,0 & 2,0 & 100 \\
\hline Prácticas de campo & 72,3 & 19,8 & 4,0 & 3,0 & 1,0 & 100 \\
\hline Autoevaluación & 40,6 & 29,7 & 20,8 & 5,9 & 3,0 & 100 \\
\hline Evaluación colectiva & 36,6 & 28,7 & 21,8 & 8,9 & 4,0 & 100 \\
\hline
\end{tabular}

\section{Fuente: elaboración propia}

La información que presenta la tabla da cuenta que las actividades menos consideradas para asignar una calificación por parte de los docentes son: examen oral $(22,8 \%)$, conducta $(34,7 \%)$, planos y maquetas $(30,7 \%)$, autoevaluación $(40,6 \%)$ y evaluación colectiva $(36,6 \%)$. Esta situación presenta dos cuestiones: la primera, que los docentes se agencian de más criterios en el momento de asignar un número; la segunda, que la autoevaluación y la evaluación colectiva aun no es sólida en la formación de los estudiantes. Se priorizan los criterios de examen escrito $(90,1 \%)$, participación en clase $(86,1 \%)$ y trabajos $(84,2 \%)$. Si bien los estudiantes llevan a cabo prácticas de campo - jornadas de observación y práctica docente-, son consideradas, pero no son las que vertebran el numeral en los estudiantes.

\section{Discusión de resultados}

La discusión de resultados que aquí se presenta, centra el análisis en la opinión que tienen los estudiantes acerca de sus hábitos de estudio y de la práctica docente de los profesores que los forman. A partir de ello se muestra cómo el hábito de estudio se debe en gran parte a la práctica docente del profesor formador. No obstante, la práctica docente refiere a todas las actividades didácticas del profesor para que el estudiante se apropie de los contenidos escolares establecidos en el plan de estudio.

\subsection{Hábitos de estudio}

Los hábitos de estudio que tienen los estudiantes se apega a los lineamientos institucionales que establece el artículo Tercero Constitucional (Constitución Política de los Estados Unidos Mexicanos, 2016) para las escuelas normales, dado que el Ejecutivo Federal delinea los planes y programas de estudio. En el hecho se cumple, el $79,2 \%$ de los estudiantes respondieron que tienen como su principal fuente de formación en Educación Especial a la antología de cada asignatura del plan de estudio.

La antología como principal fuente de lectura en los estudiantes llevó a considerar el planteamiento de Bourdieu y Passeron (1996), que los estudiantes estén condenados a recibir la visión del mundo universitario; explícitamente los profesores se sujetan al plan y programa de estudio. Se forma al estudiante para que responda al planteamiento de la política educativa nacional desde una perspectiva homogénea, que obvia las diversidades que circundan la cotidianidad del estudiantado que llega a formarse como licenciado en Educación Especial a la Escuela Normal. Se impone al estudiante un conocimiento adecuado a las relaciones objetivas del mundo académico (Bourdieu, 2009).

Al preguntarles a los estudiantes sobre los lugares donde realizan las actividades — tareas- 
que los profesores establecen para reforzar los contenidos, el $87,1 \%$ sostuvo que la casa es el principal lugar, seguido del aula de clase: el $31,7 \%$ dijo que siempre y el $40,6 \%$ que casi siempre. Los datos muestran que el aula de clase es el espacio donde se confronta el conocimiento común con el conocimiento especializado de la formación profesional, la biblioteca que debería ser el espacio donde se fortalecen esos conocimientos, a partir de búsquedas e indagación de información es minimizada por los estudiantes, dado que de acuerdo con los resultados, el 7,9\% siempre ocupa la biblioteca para hacer tareas y el $36,6 \%$ que casi siempre la utiliza.

Los tres espacios: casa, aula, biblioteca, permiten deducir que las actividades académicas - tareas- de los estudiantes se enfocan a la antología que estudian de cada asignatura, es la base de las actividades. Esta situación permite plantear la siguiente interrogante, ¿cómo generan los docentes que forman a los estudiantes de la licenciatura en educación especial, equidad y justicia en el acto educativo (Chirinos y Padrón, 2010)? $\mathrm{Al}$ pensar el cuestionamiento anterior desde las ideas de Barbosa y Moura (2013) sobre la gestión del conocimiento a partir de proyectos de trabajo, se cuestiona ¿cómo está pensando las actividades didácticas cada profesor para que el estudiante se apropie del contenido de la asignatura?

Así entonces, los hábitos de estudio de los estudiantes al centrarse en la casa y en el aula, poseen capital simbólico de alto valor (Bourdieu y Passeron, 1996). El aula, es el espacio para aprender conocimiento especializado de la formación y la casa donde reforzarlo a través de las tareas. Las consideraciones sobre la realización de actividades académicas en la casa principalmente y en segundo lugar en el aula, da cuenta que no hay formación para el uso de la biblioteca. El internet en su uso extensivo actualmente en la vida diaria de los estudiantes dentro de la Escuela Normal pudiera ser el principal factor que reduce tiempo de búsqueda de información que en la biblioteca al estudiante le consumiría más tiempo, sin embargo, solo es un supuesto hipotético, dado que el mundo fluctuante de la información, no garantiza que el estudiante se apropie de los saberes académicos de su formación que en la red digital circulan.

El hábito de estudio en los estudiantes genera otras interrogantes ¿para ellos qué es la formación en educación especial? y ¿cómo la piensan? Preguntas que en estos momentos no se responden, dado que se requiere de otro estudio. Sin embargo, el panorama que da conocer sus hábitos de estudio permite considerar que existe la preocupación de la formación, pero, limitada a la antología. Aunque en el Plan de Estudios (2004) esté el planteamiento de que el estudiante al egresar de la licenciatura contará con la siguiente habilidad intelectual:

Tiene disposición, capacidades y actitudes propias de la investigación científica: curiosidad, creatividad, capacidad de observación, habilidad para plantear preguntas metódicamente y poner a prueba respuestas, capacidad para registrar, sistematizar e interpretar información; también lleva a cabo una reflexión crítica sobre la práctica docente, y aplica estas capacidades y actitudes para mejorar los resultados de su labor educativa (Plan de Estudios, 2004, p. 45).

El planteamiento del perfil de egreso dimensiona/ambiciona un profesional de la Educación Especial con habilidades intelectuales de investigación científica. Posicionamiento contundente si se considera que la profesión docente se relaciona con teoría-práctica. Sin embargo a partir de los hábitos de estudio que aquí se presentan de los estudiantes que participaron en la investigación, se cuestiona, ¿qué elementos teóricos y metodológicos valora y pone en juego en los ensayos académicos el estudiante después de las jornadas de observación y práctica docente en cada semestre? El cuestionamiento anterior se relaciona con las habilidades de aprendizaje que el estudiante usa al leer un texto académico. El 94,1\% de los estudiantes sostuvo que elabora resúmenes, y el 79,2\% esquemas.

Generarse conocimiento académico es algo que los estudiantes han venido construyendo a 
partir de las demandas escolares que cada profesor establece. Si bien la acción del profesor formador lleva violencia simbólica como plantea Bourdieu y Passeron (1996), es en esa construcción de conocimiento donde el estudiante muestra dedicación, la cual refiere a la creencia del saber que se le forma, dado que "la creencia, pues, es constitutiva de la pertenencia a un campo" (Bourdieu, 2007, p. 109). En este caso, va inmerso el empeño, tiempo, dedicación entre otros, que el estudiante invierte para hacerse del conocimiento, tal como un artesano (Sennett, 2008), donde rompe con el conocimiento común que su medio le provee, para hacerse del saber académico (Charlot, 2008). Cabe reconocer que los estudiantes en este proceso de hacerse del conocimiento académico, desarrollan habilidades, capacidades, actitudes, entre otros, para ejercer la profesión, independientemente se acerquen o no, con el perfil de egreso que el plan de estudio establece.

Crear el conocimiento, es asumir que "las escuelas tienen que ser capaces de responder a las necesidades de los jóvenes y a facilitarles un puerto seguro en el que puedan lograrse el aprendizaje y el éxito ininterrumpidos" (Day, 2005, p. 243). Ante este planteamiento, los profesores que forman, dan margen para que "los alumnos reciban conocimientos, respondan a ellos y participen activamente en su producción" (Day, 2005, p. 249). Resulta necesario - para otro estudioinvestigar la reflexión y la investigación que el profesor formador se genera a partir de su enseñanza.

\subsection{La práctica docente que realizan los profesores formadores}

El estudiante de la licenciatura en Educación Especial ha creado una opinión de la práctica docente del profesor formador. Con base a este planteamiento se analizan las opiniones que tienen de la enseñanza de los profesores: estrategia didáctica, revisión de trabajos, asignación de calificaciones.

Respecto a las estrategias didácticas que el profesor utiliza para dar su clase, el 75,2\% de los estudiantes indicó que está centrada a que expongan, seguida de cuestionamientos $(74,3 \%)$. Esto da cuenta de un acercamiento a la dialogicidad en la construcción del conocimiento. Sin embargo, tal construcción se limita a la oferta de plan y programa de estudio. Si bien hay un planteamiento de dialéctica por parte del profesor en el reconocimiento de qué ha aprehendido el estudiante del contenido abordado, se vigila que se cumpla como se establece en el programa.

Hay un proceso áulico de reconocimiento del dominio de la teoría desde una exposición y el docente refuerza esos conocimientos con cuestionamientos para romper la frontera de saberes comunes, invitando a la reflexión, a pensar más allá del sentido común, poniéndolo en duda. Hay una gestión del conocimiento, que tiene como fin, formar docentes conocedores de su hacer. Tardif (2004) al respecto menciona que la formación inicial docente pretende habituar a los estudiantes a la práctica profesional y hacer de ellos prácticos reflexivos.

No obstante, al ser la exposición del estudiante en clase y la intervención docente la principal estrategia didáctica para la formación del conocimiento especializado, se infiere que parte de esas prácticas las están llevando a las jornadas de observación y práctica docente los estudiantes, por lo tanto, ¿cómo están llevando a cabo los estudiantes las jornadas de observación y práctica docente?, ¿repiten los ejemplos de enseñanza practicados por sus profesores?

En cuanto a la revisión de trabajos hacia los estudiantes por parte de los profesores, $24.8 \%$ sostuvo que casi nunca los profesores regresan trabajos, esto derivó los siguientes cuestionamientos ¿cuáles son esas correcciones que los docentes devuelven a los $39.6 \%$ de estudiantes que señalaron que los profesores regresan los trabajos con correcciones y comentarios?, ;el alumno atiende los comentarios?, ¿qué contenidos académicos ponderan los profesores como aspectos prioritarios a mejorar de las tareas escolares solicitadas?

La acción de los profesores de devolver trabajos con comentarios a los estudiantes se con- 
sidera favorable para el aprendizaje. El estudiante aprende de sus faltas. En el caso de estos estudiantes, con las observaciones que los profesores hacen a los trabajos que presentan, ¿qué se les fortalece académicamente para la presentación de los ensayos académicos?. El desempeño docente que el estudiante observe en clase, inciden en él al realizar el quehacer docente, dado que el ejemplo como la principal fuente de enseñanza es decisiva en la formación, pues "los docentes, al igual que otros profesionales, elaboran marcos de referencia de premisas indiscutidas que aportan orden y continuidad a su trabajo y les permiten afrontar y superar las exigencias cotidianas" (Day, 2005, p. 11). Con esas bases, el estudiante se va asumiendo como el nuevo profesionista en la docencia de la educación especial, o parafraseando a BarrónTirado y Pontón-Ramos (2013), en la escuela normal la formación pedagógica está encima del saber disciplinar.

No obstante, con las acciones de presentar el curso como mencionó $71.3 \%$ de los estudiantes y respetar las formas de evaluación acordadas como dijeron $74.3 \%$, al estudiante se le forma con el compromiso de la enseñanza. En tal compromiso converge una homogeneización de enseñanza, la cual puede ser reproducida por el estudiante en el momento de realizar las jornadas de observación y práctica docente. En este sentido, presentar el programa y acordar la formas de evaluar son rituales de los docentes al iniciar un semestre en la escuela normal.

Formarse para la docencia es asumir el compromiso que al aula se llega a cumplir el programa de estudio, independientemente del nivel escolar que se atienda, en el caso de la formación en la Educación Especial, los estudiantes han comprendido que los profesores deben dejar claras las reglas de juego al inicio del semestre, a la vez tener claro, qué juego se jugará. Los rituales que se encuentren en la práctica docente del formador, va a encontrar eco en el estudiante. Como plantea Jackson "un aspecto final de la estabilidad experimentada por los jóvenes alumnos es la calidad ritualista y cíclica de las actividades realizadas en el aula" (1996, p. 48). Se reconoce entonces, que los ejemplos son los que van acentuándose en la vida de cada uno de los que se forman.

Finalmente la asignación de calificaciones es un elemento que permea en el aprendizaje de los estudiantes, $90.1 \%$ señaló como siempre que el examen escrito es la principal herramienta de la cual hacen uso los profesores para asignar un númeral, seguido de participación en clase con $86.1 \%$ y trabajos con $84.2 \%$. A partir de las respuestas que dieron los estudiantes sobre cómo asignan un número los profesores, se llega al análisis de que la cultura de evaluación que se da a los estudiantes no prioriza la autoevaluación ni la evaluación colectiva. Desde la información proporcionada, se considera que hay un doble discurso de la evaluación, por un lado, el Plan de estudio (2004) para la formación docente en Educación Especial alude a la autoevaluación, donde el estudiante reconozca sus logros en el proceso formativo, por otro, está lo dado, el hecho de que sea una evaluación (examen) escrita la que vertebre como articuladora del conocimiento adquirido por el estudiante en el transcurso del semestre.

Profundizando en las prácticas de evaluación para la asignación de un número, estas prácticas de los docentes encuentran cabida, pues los estudiantes al egresar de la Escuela Normal se someterán a un examen para el ingreso al servicio profesional docente (Ley General del Servicio Profesional Docente, 2013). A partir de esas prácticas se sostiene la idea de que la escuela discrimina quienes sí y quiénes no caben en el Sistema Educativo Mexicano.

Las prácticas de asignación de calificación en los estudiantes se acentúan en la experiencia escolar que tienen de la formación. Tales prácticas aunque pudieran estar desvinculadas con las posturas teóricas que revisan sobre qué es la evaluación, son las que mejor se inscriben en la memoria de cada estudiante. En este caso, el que los estudiantes designen la prioridad de sus profesores por el examen escrito, da cuenta que es la práctica más usual en la didáctica de ellos para 
obtener un parámetro de conocimiento respecto a la especialización en la profesión.

El examen escrito al ser parámetro de numeral, somete a estudiantes y docentes sobre qué se debe aprender, cómo se debe hacer y qué se tiene que responder. Es una camisa de fuerza que limita una autonomía del profesor en formación (Contreras, 1997). De tal forma que se le prepara para responder a los parámetros que el Sistema Educativo considera como los ideales.

\section{Conclusiones}

En el manuscrito se ha planteado y discutido sobre los hábitos de estudio de los estudiantes de la licenciatura en Educación Especial y la opinión que tienen de la práctica docente de los profesores que los forman. Se reconoce, a partir de los resultados dados por los estudiantes, que la principal preocupación que tienen los profesores hacia ellos, es que consuman en su totalidad la teoría que se plantea en las antologías de cada asignatura que se ha establecido desde el Sistema Educativo. De ahí que derive que los hábitos de estudio que dicen tener, considere al resumen y a los esquemas, como las prioritarias para atender el contenido de una lectura, a la vez, situar el espacio de la casa como el lugar donde organizan sus ideas para atender los planteamientos de cada asignatura. Con base a esa organización de ideas sistematizadas construyen los trabajos académicos que solicitan los profesores y participan individual o grupalmente en la clase. A la vez, el saber académico que construyen en un periodo del semestre, se mostrará al profesor a través del examen escrito.

Así entonces, los hábitos de estudio de estos estudiantes, cultivan el saber responder a lo que se pide. En ello van inmersas las prácticas docentes de los profesores, quienes priorizan el cumplimiento del programa de estudio, que el ejemplo de enseñanza que muestran al estudiante -desde la práctica docente que ejercen- es el de saber atender los propósitos del programa académico. En este caso, las prácticas docentes que los alumnos han ido incorporando de sus profesores es que hay que encuadrar el desarrollo de la asignatura, generar dispositivos como: participación en clase, entrega de trabajos, exposición grupal, exposición individual y hacer examen escrito. Prácticas que los profesores formadores realizan en la cotidianidad escolar, una enseñanza sistematizada que se hereda al alumno a través del proceso formativo. Se hace de la enseñanza a través del ejemplo, la disciplina con la que se interviene en el quehacer docente.

Finalmente, los resultados encontrados respecto a los hábitos de estudio de los estudiantes con los que enfrentan el plan de estudio de cada asignatura, les ha permitido hacerse del saber académico, más la interiorización que han hecho de las prácticas docentes de los profesores que los forman, les va dando elementos para comprender qué es formarse como docente en Educación Especial.

\section{Referencias bibliográficas}

Álvarez, C., Silió, G. \& Fernández, E. (2012).Planificación, colaboración, innovación: tres claves para conseguir una buena práctica docente universitaria. Revista de Docencia Universitaria. REDU, 10(1), 415-430. Recuperado de https://bit.ly/2rfdXyx

Barbosa, E. F., \& Moura, D. G. (2013). Proyectos educativos y sociales. Planificación, gestión, seguimiento y evaluación. Madrid: Narcea.

Barrón-Tirado, C., \& Pontón-Ramos, C. (2013). La reforma de la Escuela Normal de 1997. Algunas consideraciones críticas. En P. Ducoing Watty, La escuela normal: una mirada desde el otro (pp. 191-222). México: UNAM.

Bericat, A. E. (1998). La integración de los métodos cuantitativo y cualitativo en la investigación social: Significado y medida. Barcelona: Editorial Ariel.

Bourdieu, P. (2007). El sentido práctico. Buenos Aires: Siglo XXI Editores.

Bourdieu, P. (2009). Homo academicus. México: Siglo Veintiuno.

Bourdieu, P., \& Passeron, J. C. (1996). La reproducción. Elementos para una teoría del sistema de enseñanza. México: Fontamara.

Campos, A. (2014). Métodos mixtos de investigación: Integración de la investigación cuantitativa y la investigación cualitativa. Bogotá: Magisterio. 
Charlot, B. (2008). La relación con el saber, formación de maestros y profesores, educación y globalización. Cuestiones para la educación de hoy. Montevideo: TRILCE.

Constitución Política de los Estados Unidos Mexicanos. (2016). Cámara de Diputados. Diario Oficial de la Federación.

Contreras, J. (1997). La autonomía del profesorado. Madrid: Morata.

Chirinos, N. y Padrón, E. (2010). La eficiencia docente en la práctica educativa. Revista de Ciencias Sociales (Ve), XVI (3), 481-492. Recuperado de https://bit.ly/2FyWG7G

Day, C. (2005). Formar docentes. Cómo, cuándo y en qué condiciones aprende el profesorado. Madrid: Narcea.

Escalante, L., Escalante, Y., Linzaga, C., \& Merlos, M. (2008). Comportamiento de los estudiantes en función a sus hábitos de estudio. Revista Electrónica "Actualidades Investigativas en Educación", 8 (2), 1-15. Recuperado de https://bit.ly/2rextKF.

Farías-Martínez, G. M., \& Ramírez-Montoya, M. S. (2010). Desarrollo de cualidades reflexivas de profesores en formación inicial a través de portafolios electrónicos. Revista Mexicana de Investigación Educativa , 15 (44), 141-162. Recuperado de https://goo.gl/IfVjEh.

Gallego-Ortega, J. L., \& Rodríguez-Fuentes, A. (2007). Tendencias en la formación inicial del profesorado en educación especial. Revista Iberoamericana sobre Calidad, Eficacia y Cambio en Educación, 5 (3), 102-117. Recuperado de https://goo.gl/IfVjEh.

Jackson, P. (1996). La vida en las aulas. Madrid: Morata.

Ley General de Educación. (2013). Ley General de Educación. Última Publicación 20/05/2014. México: Cámara de Diputados del $\mathrm{H}$. Congreso de la Unión.

Ley General del Servicio Profesional Docente. (2013). México: Diario Oficial de la Federación.
Ortiz, F. G., \& García, M. P. (2016). Metodología de la investigación: El proceso y sus técnicas. México: Limusa.

Plan de Estudios. (2004). Licenciatura de Educación Especial. Programa para la transformación y el Fortalecimiento Académicos de las Escuelas Normales. México: Secretaría de Educación Pública.

Plan Nacional de Desarrollo. (2013). Plan Nacional de Desarrollo 2013-2018. Gobierno de la República.

Programa Sectorial de Educación. (2013). Programa Sectorial de Educación, 2013-2018. Secretaría de Educación Pública .

Sánchez-Palomino, A. (2007). Investigación sobre la formación inicial del profesorado de educación secundaria para la atención educativa a los estudiantes con necesidades especiales. Revista Interuniversitaria de Formación del Profesorado, 21 (2-3), 149-181. Recuperado de https://goo.gl/78yP4Y.

Secretaría de Educación Pública (2006). Orientaciones generales para el funcionamiento de los servicios de educación especial. México: SEP.

Secretaría de Educación Pública (2010). Memorias y actualidad en la Educación Especial de México: Una visión histórica de sus modelos de atención. México, D. F. SEP, Dirección General de Educación Especial

Sennett, R. (2008). El artesano. Barcelona: Anagrama. Tardif, M. (2004). Los saberes del docente y su desarrollo profesional. Madrid: Narcea.

Tenorio, S. (2011). Formación inicial docente $y$ necesidades educativas especiales. Estudios Pedagógicos, XXXVII (2), 249-265. Recuperado de https://goo.gl/Ww42cf.

Torres, M., Tolosa, I., Urrea, M., \& Monsalve, A. (2009). Hábitos de estudio vs. Fracaso académico. Revista Educación, 33 (2), 15-24. Recuperado de https://bit.ly/2jjZCwe. 